\title{
Analytical Method of Forming the Equivalent Virtual MIMO Channel Matrix for Space-Time Codes of High Dimensions
}

\author{
Andrey Reznev \\ Scientific and Research Center ATLAS \\ Moscow, Russia \\ Andrey.Reznev@gmail.com
}

\begin{abstract}
This work provides the analytical method for Equivalent Virtual Channel Matrix (EVCM) synthesis. The approach of Equivalent Virtual Channel Matrix might be applied for any type of STC to convert it into BLAST STC structure. The work demonstrates that manual methods are not applicable for large scale STC structures due to overladen manual derivation. Decomposition of STC codes matrices into multiplication product of channel part and symbols allows picking the symbol vector as the separate part of model equation. Linear transformation converts the multiplication product into BLAST type system with new channel matrix. This virtual channel matrix equation is the analytical equation for EVCM.
\end{abstract}

Key words: MIMO, BLAST, EVCM, Equivalent Virtual Channel Matrix, Space Time Coding, Golden code, STC.

\section{INTRODUCTION}

The group of technologies of high speed mobile Internet access is coming, the marketing umbrella for those ones is "Fifth Generation - 5G". Smooth migration from 4G to 5G supported by radio access technology named Multiple Input - Multiple Output (MIMO), which uses the number of transmitting and receiving antennas within the system. Some of the 3GPP standards declare that possible numbers of antennas may exceed 128 [1].

The necessity to increase number of antennas provides by BLAST which is the common space time coding approach, when one information symbol sends through one antenna simultaneously with other symbols [2;3]. One of the ways is transmission for several time intervals which might decrease the number of antennas in MIMO.

\section{SYSTEM MODEL}

Some of the well-known multi-interval STCs might be orthogonal in order to achieve low decoding complexity [2].

Alamouti and Golden structures are STC which use multiinterval transmission, and they both need to be transformed into BLAST system model. The way of transformation replaces the channel matrix to Equivalent Virtual Channel Matrix (EVCM) [2], which is pretty simple for two antennas but requires the overladen manual works for the big MIMO system.

EVCM structure is the composition of channel information and coding method with the dimension which is the result related to number of transmission antennas and transmission intervals.
In case of Alamouti STC, we faced with the system of two equations which give us $2 \times 2$ matrix, for classical Golden we can see the system of four equations and $4 \times 4$ matrix. The $A B$ or ABBA combination will bring us at least eight complicated equations with $8 \times 8$ matrixes. The increased numbers of antennas is the result of Shannon-Hartley theorem when the system throughput multiplies with numbers of paths between transmitting and receiving antennas. 5G speed levels will accelerate the number of antennas $[2 ; 4]$.

We use system model, see (1) Ошибка! Источник ссылки не найден.; [3]:

$$
\mathbf{Y}=\mathbf{H} \cdot \mathbf{S}(\boldsymbol{\theta})+\boldsymbol{\Gamma}
$$

In the system model we have: $\mathbf{Y}$ is received signal matrix, dimension is $M \times L ; \mathbf{H}$ is channel matrix, dimension $M \times N$; $\mathbf{S}(\boldsymbol{\theta})$ is space time code matrix, dimension $N \times L ; \boldsymbol{\theta}$ is transmitted information vector, dimension $Q \times 1 ; \boldsymbol{\Gamma}$ is an independent, identically distributed noise matrix, dimension $M \times L ; M$ is number of receiving antennas; $N$ is number of transmitting antennas; $L$ is number of time intervals for transmitting; $Q$ is number of transmitting information symbols in STC.

Some of the STCs may include symbols and complex conjugate symbols both, the typical example is Alamouti code which is the origin code for numbers of large STCs. We need to consider this to produce correct common method for EVCM synthesis. We generalize the system model (1) to the new equation to add complex conjugate symbols (2):

$$
\mathbf{Y}=\mathbf{H} \cdot \mathbf{S}\left(\boldsymbol{\theta}, \boldsymbol{\theta}^{*}\right)+\boldsymbol{\Gamma}
$$

\section{PROPOSED METHOD AND RESULTS}

For STC we may have following view (3), where every matrix element depends on the whole raw of information symbols including their conjugate values:

$$
\mathbf{S}\left(\boldsymbol{\theta}, \boldsymbol{\theta}^{*}\right)=\left[\begin{array}{ccc}
S_{11}\left(\theta_{1}, \theta_{2} \ldots \theta_{Q} ; \theta_{1}^{\prime}, \theta_{2}^{\prime} \ldots \theta_{Q}^{\prime}\right) & \cdots & S_{1 L}\left(\theta_{1}, \theta_{2} \ldots \theta_{Q} ; \theta_{1}^{\prime}, \theta_{2}^{\prime} \ldots \theta_{Q}^{\prime}\right) \\
\vdots & \vdots & \vdots \\
S_{N 1}\left(\theta_{1}, \theta_{2} \ldots \theta_{Q} ; \theta_{1}^{\prime}, \theta_{2}^{\prime} \ldots \theta_{Q}^{\prime}\right) & \cdots & S_{N L}\left(\theta_{1}, \theta_{2} \ldots \theta_{Q} ; \theta_{1}^{\prime}, \theta_{2}^{\prime} \ldots \theta_{Q}^{\prime}\right)
\end{array}\right]
$$


Now every element $S_{i, j}\left(\theta_{1}, \theta_{2} \ldots \theta_{Q} ; \theta_{1}^{\prime}, \theta_{2}^{\prime} \ldots \theta_{Q}^{\prime}\right)$ of matrix $\mathbf{S}\left(\boldsymbol{\theta}, \boldsymbol{\theta}^{*}\right)$ (3) might be represented as (4):

$$
\begin{aligned}
& \quad S_{i, j}\left(\theta_{1}, \theta_{2} \ldots \theta_{Q} ; \theta_{1}^{\prime}, \theta_{2}^{\prime} \ldots \theta_{Q}^{\prime}\right)=\sum_{q=1}^{Q} \alpha_{i, j} \cdot \theta_{q}+\beta_{i, j} \cdot \theta_{q}^{\prime} \\
& i=1 ; 2 ; \ldots N, j=1 ; 2 ; \ldots L
\end{aligned},
$$

Those $\alpha_{i, j}(q), \beta_{i, j}(q)$ factors inherit the original STC properties.

Combining (4), (3), we see (5):

$$
\begin{aligned}
& \mathbf{S}\left(\boldsymbol{\theta}, \boldsymbol{\theta}^{*}\right)=\left[\begin{array}{ccc}
\sum_{q=1}^{Q} \alpha_{11}(q) \cdot \theta_{q}+\beta_{11}(q) \cdot \theta_{q}^{\prime} & \cdots & \sum_{q=1}^{Q} \alpha_{1 L}(q) \cdot \theta_{q}+\beta_{1 L}(q) \cdot \theta_{q}^{\prime} \\
\vdots & \vdots & \vdots \\
\sum_{q=1}^{Q} \alpha_{N 1}(q) \cdot \theta_{q}+\beta_{N 1}(q) \cdot \theta_{q}^{\prime} & \cdots & \sum_{q=1}^{Q} \alpha_{N L}(q) \cdot \theta_{q}+\beta_{N L}(q) \cdot \theta_{q}^{\prime}
\end{array}\right]= \\
& =\sum_{q=1}^{Q}\left[\begin{array}{ccc}
\alpha_{11}(q) & \cdots & \alpha_{L L}(q) \\
\vdots & \vdots & \vdots \\
\alpha_{N 1}(q) & \cdots & \alpha_{N L}(q)
\end{array}\right] \cdot \theta_{q}+\left[\begin{array}{ccc}
\beta_{11}(q) & \cdots & \beta_{L L}(q) \\
\vdots & \vdots & \vdots \\
\beta_{N 1}(q) & \cdots & \beta_{N L}(q)
\end{array}\right] \cdot \theta_{q}^{\prime}
\end{aligned}
$$

Reorganize (5) to the compact view (6):

$$
\begin{gathered}
\mathbf{S}\left(\boldsymbol{\theta}, \boldsymbol{\theta}^{*}\right)=\sum_{q=1}^{Q} \mathbf{A}_{q} \cdot \theta_{q}+\mathbf{B}_{q} \cdot \theta_{q}^{\prime}, \text { where } \\
\mathbf{A}_{q}=\left[\begin{array}{ccc}
\alpha_{11}(q) & \cdots & \alpha_{1 L}(q) \\
\vdots & \vdots & \vdots \\
\alpha_{N 1}(q) & \cdots & \alpha_{N L}(q)
\end{array}\right], \\
\mathbf{B}_{q}=\left[\begin{array}{ccc}
\beta_{11}(q) & \cdots & \beta_{1 L}(q) \\
\vdots & \vdots & \vdots \\
\beta_{N 1}(q) & \cdots & \beta_{N L}(q)
\end{array}\right]_{q=1 ; 2 ; \ldots Q}
\end{gathered}
$$

New matrices $\mathbf{A}_{q} \mathbf{B}_{q}$ derived from original STC structure. We might use our preparations to transform the signal model to virtual BLAST type system (2).

For our further steps we present the transmitted symbols to make explicit real and imaginary parts (7):

$$
\begin{aligned}
& \theta_{q}=\operatorname{Re}\left(\theta_{q}\right)+j \operatorname{Im}\left(\theta_{q}\right)=\theta_{R q}+j \theta_{I q} \\
& \theta_{q}^{\prime}=\operatorname{Re}\left(\theta_{q}^{\prime}\right)-j \operatorname{Im}\left(\theta_{q}^{\prime}\right)=\theta_{R q}-j \theta_{I q}
\end{aligned}
$$

Combining (7) and (6), we see (8):

$$
\begin{aligned}
& \mathbf{S}\left(\boldsymbol{\theta}, \boldsymbol{\theta}^{*}\right)=\sum_{q=1}^{Q} \mathbf{A}_{q}\left(\theta_{R q}+j \theta_{I q}\right)+\mathbf{B}_{q}\left(\theta_{R q}-j \theta_{I q}\right)= \\
& =\sum_{q=1}^{Q}\left(\mathbf{A}_{q}+\mathbf{B}_{q}\right) \cdot \theta_{R q}+j\left(\mathbf{A}_{q}-\mathbf{B}_{q}\right) \cdot \theta_{I q}
\end{aligned}
$$

Apply vectorization to model (2),

$$
\mathbf{Y}=(\mathbf{1} \otimes \mathbf{H}) \cdot \operatorname{vec}\left(\mathbf{S}\left(\boldsymbol{\theta}, \boldsymbol{\theta}^{*}\right)\right)+\boldsymbol{\eta}
$$

Vectorization result for STC has the new form (10):

$$
\begin{aligned}
& \operatorname{vec}\left(\mathbf{S}\left(\boldsymbol{\theta}, \boldsymbol{\theta}^{*}\right)\right)=\operatorname{vec}\left(\sum_{q=1}^{Q}\left(\mathbf{A}_{q}+\mathbf{B}_{q}\right) \cdot \theta_{R q}+j\left(\mathbf{A}_{q}-\mathbf{B}_{q}\right) \cdot \theta_{I q}\right)= \\
& =\sum_{q=1}^{Q} \theta_{R q} \cdot \operatorname{vec}\left(\mathbf{A}_{q}+\mathbf{B}_{q}\right)+j \theta_{I q} \cdot \operatorname{vec}\left(\mathbf{A}_{q}-\mathbf{B}_{q}\right)
\end{aligned}
$$

Let's mark some variables within (10) and combine multipliers, and see (11):

$$
\begin{aligned}
& \operatorname{vec}\left(\mathbf{S}\left(\boldsymbol{\theta}, \boldsymbol{\theta}^{*}\right)\right)=\mathbf{T}_{1} \cdot \boldsymbol{\theta}_{R}+j \cdot \mathbf{T}_{2} \cdot \boldsymbol{\theta}_{I}, \text { where } \\
& \mathbf{T}_{1}=\left[\operatorname{vec}\left(\mathbf{A}_{1}+\mathbf{B}_{1}\right) ; \operatorname{vec}\left(\mathbf{A}_{2}+\mathbf{B}_{2}\right) \ldots v e c\left(\mathbf{A}_{Q}+\mathbf{B}_{Q}\right)\right] \\
& \mathbf{T}_{2}=\left[\operatorname{vec}\left(\mathbf{A}_{1}-\mathbf{B}_{1}\right) ; \operatorname{vec}\left(\mathbf{A}_{2}-\mathbf{B}_{2}\right) \ldots \operatorname{vec}\left(\mathbf{A}_{Q}-\mathbf{B}_{Q}\right)\right] \\
& \boldsymbol{\theta}_{R}=\left|\begin{array}{c}
\theta_{R 1} \\
\theta_{R 2} \\
\vdots \\
\theta_{R Q}
\end{array}\right|, \\
& \boldsymbol{\theta}_{I}=\left|\begin{array}{c}
\theta_{I 2} \\
\vdots \\
\theta_{I Q}
\end{array}\right|
\end{aligned}
$$

Substituting (11) in (9), we can get the new signal model (12):

$$
\mathbf{Y}=(1 \otimes \mathbf{H}) \cdot\left(\mathbf{T}_{1} \cdot \boldsymbol{\theta}_{R}+j \mathbf{T}_{2} \boldsymbol{\theta}_{I}\right)+\boldsymbol{\eta}
$$

In order to simplify the equation we may reorganize to (12) to

$$
\mathbf{Y}=(1 \otimes \mathbf{H}) \cdot \mathbf{J} \cdot \boldsymbol{\theta}^{R / I}+\boldsymbol{\eta} \text {, where }
$$




$$
\mathbf{J}=\left[\mathbf{T}_{1} ; j \mathbf{T}_{2}\right]
$$

$\boldsymbol{\theta}^{R / I}=\left|\begin{array}{l}\boldsymbol{\theta}^{R} \\ \boldsymbol{\theta}^{I}\end{array}\right|=\left|\begin{array}{llllllll}\theta_{1}^{R} & \theta_{2}^{R} & \ldots & \theta_{Q}^{R} & \theta_{1}^{R} & \theta_{1}^{R} & \ldots & \theta_{1}^{R}\end{array}\right|^{\mathrm{T}}$

Analytical equation for EVCM has the view.

$$
\mathbf{H}_{E V C M}=(\mathbf{1} \otimes \mathbf{H}) \cdot \mathbf{J}
$$

EVCM system model transformation for information sequences with complex conjugate symbols doubling the size of EVCM dimension and transmitting vector should be replaced to double size vector constructed from real and imaginary parts of original symbols.

\section{REFERENCES}

[1] TS 23.501. System Architecture for the 5G System. ETSI. 2017.

[2] Бакулин М.Г., Варукина Л.А., Крейнделин В.Б. Технология MIMO: принципы и алгоритмы. М.: Горячая линия - Телеком, 2014 [Bakulin M.G., Varukina L.A., Kreyndelin V.B. MIMO Technology: Principles and Algorithms. Moscow: Hotline-Telecom, 2014].

[3] Larsson E. G., Stoica P. Space-Time Block Coding for Wireless Communications. $3^{\text {rd }}$ ed. Cambridge: Cambridge University Press, 2008.

[4] Oesges C., Clerckx B. MIMO Wireless Communications. Channels, Techniques and Standards for Multi-Antenna, Multi-User and MultiCell Systems. Oxford: Academic Press, 2013.

[5] Крейнделин В., Резнев А. Методы формирования пространственно временных матриц для систем МIMО высокой размерности // Электросвязь. 2017. №4. С. 46-50. [Kreindelin V.B., Reznev A.A. Methods for STC matrices synthesis for Large Scale MIMO structures // Electrical Communications. 2017. №4. P. 46-50]. 